\title{
Inducción de la brotación in vitro de microplántulas de Nogal (Juglans neotropica) tratadas con Thidiazuron (TDZ) y 6-Bencilaminnopurina (BAP)
}

\author{
Denisse F. Peña, Melida N. Rocano, Jazmin M. Salazar, Carlos S. Torres \\ Carrera de Ingeniería Agronómica, Facultad de Ciencias Agropecuarias, Universidad de Cuenca. \\ Avenida 12 de Octubre y Diego de Tapia, Cuenca, Ecuador. \\ Autor de correspondencia: denisse.pena@ucuenca.edu.ec \\ Fecha de recepción: 8 de agosto de 2014 - Fecha de aceptación: 20 de septiembre de 2014
}

\begin{abstract}
RESUMEN
El nogal (Juglans neotropica) es una especie de gran potencial para proyectos de conservación y agroproductivos en la eco-región Andina. No obstante, su baja capacidad germinativa limita su propagación para reforestación. El objetivo de este estudio fue determinar el efecto del tratamiento con Thidiazuron (TDZ) y 6-Bencilaminopurina (BAP) en la inducción in vitro de brotes de J. neotropica. Las microplántulas tratadas con BAP produjeron más brotes que aquellas tratadas con TDZ. Las microplántulas tratadas con BAP y TDZ generaron a su vez más brotes que las no-tratadas. En el ensayo se evaluó también el efecto de la concentración de BAP y TDZ en el medio pero no se detectaron diferencias estadísticamente significativas entre las concentraciones evaluadas. En conjunto, nuestros resultados indican que el tratamiento con cito-quininas, específicamente BAP, incrementa el número de brotes producidos por microplántula de $J$. neotropica, pudiendo aplicarse este tratamiento en proyectos de propagación masiva de esta especie para sostener programas de reforestación.
\end{abstract}

Palabras clave: Tocte, cedro negro, multiplicación in vitro.

\begin{abstract}
The walnut (Juglans neotropica) is a species of great potential for conservation projects and eco-agroproduction in the Andean region. However, low germination capacity limits its spread for reforestation. The aim of this study was to determine the effect of treatment with Thidiazuron (TDZ) and 6-Benzylaminopurine (BAP) on in vitro shoot induction of $J$. neotropica. The micro plantlets treated with BAP produced more shoots than those treated with TDZ. The micro plantlets treated with BAP and TDZ in turn generated more shoots than the non-treated micro plantlets. In the test, the effect of concentration of BAP and TDZ in the medium was also evaluated; however not statistically significant differences between the tested concentrations were detected. In summary, findings indicate that the treatment with cytokines, BAP specifically, increases the number of shoots produced by micro plantlets J. neotropica. It is believed that this treatment can be applied on projects of mass propagation of this species within the frame of reforestation programs.
\end{abstract}

Keywords: Tocte, black cedar, in vitro multiplication.

\section{INTRODUCCIÓN}

La reforestación con especies nativas constituye una herramienta promisoria para la restauración de ecosistemas degradados en la región sur del Ecuador (Aguirre y col., 2007). La presión continua e intensa que ejerce la población rural sobre los recursos naturales de la eco-región Andina; es uno de los puntos centrales de preocupación en la Agenda Nacional para lograr el desarrollo sostenible (Grijalva y col., 2010). Entre las especies andinas, Juglans neotropica, es de particular importancia 
para las comunidades locales, ya que además de proporcionar cobertura vegetal, esta especie es aprovechada también por sus propiedades medicinales (Geilfus y Bailon, 1994), alimenticias e industriales (Masias Bröcker, 2007; Ponce y Morales, 2011). El nogal es una especie de tierras altas (1000-3000 m.s.n.m) y crece naturalmente desde Colombia hasta Bolivia (Mendez, 2000).

En Juglans regia la estratificación reduce las cantidades de inhibidores en las semillas resultando beneficioso para la germinación de esta especie (Martin y col., 1969). López Carvajal y Piedrahíta Cardona (1998) reportan una germinación de entre el 40-85\% para varias especies del género Juglans, pero los mismos autores, concluyen que para Juglans neotropica los tratamientos de estratificación durante 30 y 60 días no fueron eficaces para superar la latencia profunda en esta especie, sugiriendo que probablemente se requieren periodos de estratificación mayores, entre 90 y 120 días.

Debido a su baja germinación natural, los métodos de propagación asistida in vitro representan una alternativa para el abastecimiento de grandes números de plantas para la propagación de estas especies (Sánchez-Olate y col., 2009). El cultivo in vitro de embriones suprime el tiempo de estratificación sugerido por López (1999) y permite alcanzar hasta el $100 \%$ de germinación a los 15 días de cultivo (Quintero-García y col., 2012). Varios estudios sobre el desarrollo de técnicas para el cultivo in vitro del género Juglans han sido reportados (Pijut, 1997; Quintero-García y col., 2012) sin embargo pocos han sido publicados para la inducción de brotes en la especie J. neotropica.

En este artículo reportamos avances en el desarrollo de un protocolo de propagación in vitro de $J$. neotropica a través del tratamiento de microplántulas con dos cito-quininas como parte de un proyecto de propagación masiva de esta especie con fines de agro-producción y restauración.

\section{MATERIALES Y MÉTODOS}

Se colectaron semillas maduras de J. neotropica de árboles distribuidos en la cuenca del río Paute (Azuay, Ecuador). Los embriones de las semillas colectadas fueron aislados después de la ruptura mecánica de la testa de las semillas. Los embriones extraídos, aún con parte del cotiledón, fueron lavados con agua destilada estéril, y desinfectados con una solución de etanol al 70\% por 3 segundos, sin enjuague, luego de lo cual se aislaron los embriones en una cámara de flujo. Los embriones fueron a continuación sumergidos en hipoclorito de sodio al 0,07\% por 5 segundos y sembrados en medio de cultivo M\&S (Murashige y Skoog Medio Basal) (Murashige y Skoog, 1962).

Los embriones aislados fueron incubados en un cuarto de cultivo con foto-período de $16 \mathrm{~h}$ luz por treinta días. Después de la incubación las microplántulas obtenidas, que medían en promedio $4 \mathrm{~cm}$ de altura, fueron transferidas a frascos que contenían $30 \mathrm{ml}$ de medio DKW (Driver y Kuniyuki, 1984) considerando los resultados obtenidos por Bosela y Michler (2008). El medio fue suplementado con dos concentraciones 0,05 y $0,07 \mathrm{mg} \mathrm{l}^{-1}$, de 6-Bencilaminopurina (BAP) y Thidiazuron (TDZ). Los autores seleccionaron estas concentraciones en base a resultados de estudios preliminares en otras especies forestales (datos no publicados). En cada frasco se colocaron dos plantas y los frascos fueron mantenidos bajo las mismas condiciones de crecimiento descritas anteriormente por 30 días.

El experimento se condujo utilizando un diseño completamente al azar en arreglo factorial con 10 frascos por tratamiento. Se aplicó el análisis de varianza a los resultados de la prueba; la existencia de diferencias significativas se evaluó a través de la prueba de Duncan en el programa SPSS estadísticas 22.0.

\section{RESULTADOS Y DISCUSIÓN}

Bajo condiciones naturales, los embriones de J. neotropica presentan una baja capacidad de germinación y requieren de prolongados (40-60 días) tratamientos pre-germinativos para escarificar y romper la latencia del embrión (Quintero-García, 2012). En este estudio, la germinación de los embriones se registró a partir del quinto día, obteniéndose un porcentaje de germinación del $80 \%$ a los 15 días de la siembra. 
Las microplántulas tratadas con BAP produjeron más brotes que las tratadas con TDZ y sin tratamiento $(\mathrm{p}<0.05)$ (ver Tabla 1 y Fig. 1). No se registraron diferencias significativas $(\mathrm{p}=0.05)$ entre las concentraciones evaluadas. Las microplántulas no tratadas no produjeron ningún brote.

Tabla 1. Efecto de TDZ y BAP en la brotación in vitro de Juglans neotropica.

\begin{tabular}{cccccc}
\hline Tratamiento & $\begin{array}{c}\text { Índice } \\
\text { promedio }\end{array}$ & $\begin{array}{c}\text { Error } \\
\text { estándar del } \\
\text { índice }\end{array}$ & $\begin{array}{c}\text { Total de } \\
\text { brotes por } \\
\text { tratamiento }\end{array}$ & $\begin{array}{c}\text { Mínimo } \\
\text { brotes por } \\
\text { tratamiento }\end{array}$ & $\begin{array}{c}\text { Máximo } \\
\text { brotes por } \\
\text { tratamiento }\end{array}$ \\
\hline $\begin{array}{c}\text { Control } \\
\text { TDZ a } 0,05 \mathrm{mg} \mathrm{l}^{-1}\end{array}$ & $0,50 \mathrm{a}$ & 0 & 0 & 0 & 0 \\
TDZ a $0,07 \mathrm{mg} \mathrm{l}^{-1}$ & $0,60 \mathrm{a}$ & 0,21 & 9 & 0 & 4 \\
BAP a $0,05 \mathrm{mg} \mathrm{l}^{-1}$ & $2,05 \mathrm{~b}$ & 0,37 & 12 & 0 & 5 \\
BAP a $0,07 \mathrm{mg} \mathrm{l}^{-1}$ & $2,80 \mathrm{~b}$ & 0,29 & 41 & 1 & 7 \\
\hline
\end{tabular}

- Valores seguidos por una misma letra en cada columna, no difieren significativamente $(P \geq 0,05)$ de acuerdo a la Prueba de Rangos Múltiples de Duncan.
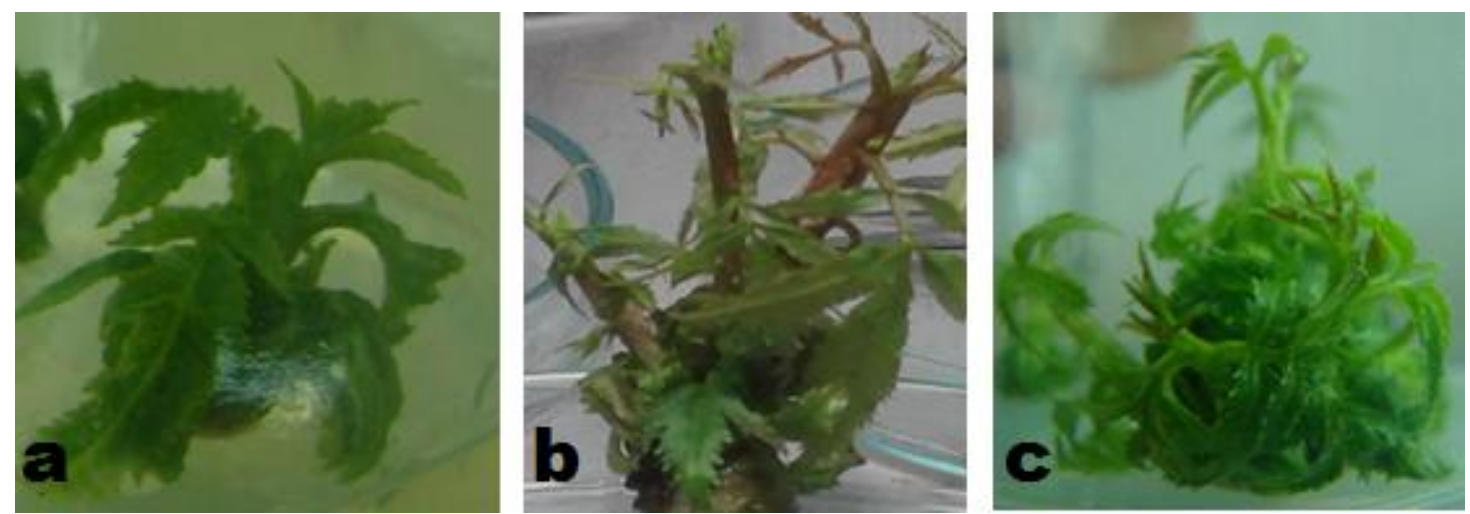

Figura 1. Brotación in vitro de Juglans neotropica: (a) Microplántula cultivada en DKW + $0.05 \mathrm{mg} \mathrm{l}^{-1}$ TDZ; (b) Microplántula cultivada en DKW + $0.05 \mathrm{mg} \mathrm{l}^{-1}$ BAP; (c) Microplántula cultivada en DKW + $0.07 \mathrm{mg} \mathrm{l}^{-1} \mathrm{BAP}$.

En cultivo de tejidos in vitro, las cito-quininas inducen la división celular y tienen un papel fundamental en el proceso de organogénesis (Hurtado y Merino, 1988; Mock, 2000). El papel de BAP como una cito-quinina eficaz en la multiplicación ha sido establecido en muchas plantas (Al-Mizory y Mayi, 2012). TDZ ha tenido también un efecto positivo (75-87\%) en la proliferación de segmentos nodales de Juglans nigra (Bosela y Michler, 2008). En este estudio, demostramos que tratando microplántulas de J. neotropica con dos cito-quininas (BAP y TDZ) se incrementa el número de brotes inducidos por microplántula. Resultados similares han sido reportados para Juglans regia, obteniéndose entre 1,22 a 2,84 brotes por microplántula bajo diferentes concentraciones de BAP y Kinetina por separado (Al-Mizory y Mayi, 2012). En nuestro estudio, el tratamiento de microplántulas con BAP estimuló la brotación en mayor medida, que el tratamiento con TDZ. Este efecto también ha sido reportado en Juglans regia L. donde $0,5 \mathrm{mg} \mathrm{l}^{-1}$ de BAP se estableció como tratamiento óptimo para la etapa de proliferación (Toosi y Dilmagani, 2010). El máximo número de brotes en microplántulas de $J$. regia también con BAP $\left(2 \mathrm{mg} \mathrm{l}^{-1}\right)$ fue reportado por Al-Mizory y Mayi (2012). Además, no detectamos diferencias en la inducción de brotes asociadas a las concentraciones de las cito-quininas evaluadas. Explorar si se obtienen efectos similares a los reportados en otras especies de Juglans a concentraciones menores de cito-quininas es útil para optimizar el proceso de propagación masiva en Juglans neotropica. 


\section{CONCLUSIONES}

El tratamiento de plantas con cito-quininas incrementa la brotación in vitro en J. neotropica. La brotación es sensible al tipo de cito-quinina utilizada, siendo BAP más eficiente que TDZ para inducir brotación en microplántulas obtenidas de embriones germinados in vitro. En este estudio no se reportaron diferencias entre los niveles de concentración de cito-quinina en el medio. Los resultados obtenidos contribuyen a la optimización del proceso de propagación in vitro de esta especie para el soporte de programas de reforestación activos en el Austro.

\section{BIBLIOGRAFÍA}

Al-Mizory, L.S.M., A.A. Mayi, 2012. In vitro propagation of walnut (Juglans regia) by Nadal Explants. JAST, B2, 665-670.

Aguirre, N., S. Günter, B. Stimm, B., 2007. Mejoramiento de la propagación de especies forestales nativas del bosque montano en el Sur del Ecuador. Descargado de http://www.rncalliance.org/ WebRoot/rncalliance/Shops/rncalliance/4C15/957A/9D0B/09EB/B5F4/C0A8/D218/8324/Aguirre _et_al_2007_mejoraiento_propagacion.pdf en enero 2014, 8 pp.

Bosela, M., C. Michler, 2008. Media effects on black walnut (Juglans nigra L.) shoot culture growth in vitro: evaluation of multiple nutrient formulations and cytokinin types. Vitro Cell. Dev. Biol.Plant, 44, 316-329.

Driver, J., A. Kuniyuki, 1984. In vitro propagation of Paradox walnut rootstock. Hortic. Sci., 19, 507509.

Geilfus, F., P. Bailon, 1994. El árbol al servicio del agricultor: manual de agroforestería para el desarrollo rural. Vol. 2. Serie técnica. Manual técnico / CATIE No. 9, 656 pp. CATIE, Turrialba, Costa Rica. Descargado de http://en.booksee.org/book/l304639 en mayo 2014.

Grijalva, J., D. Riofrío, A. Ramos, 2010. Árboles y arbustos para la conservación de microcuencas alto andinas y adaptación al cambio climático. Micro cuenca del río Chimborazo. Boletín Divulgativo No. 369, 23 pp. NINA comunicaciones. Descargado de http://www.iniap.gob.ec/nsite/images/ documentos/ Arboles\%20y\%20arbustos\%20para\%20la\%20conservaci\%C3\%B3n\%20de\%20 microcuencas\%20y\%20adaptaci\%C3\%B3n\%20al\%20cambio\%20climatico..pdf en abril 2014.

Hurtado, D.V., M.E. Merino, 1998. Cultivo de tejidos vegetales. Editorial Trillas, México, 232 pp.

López Carvajal, J., E. Piedrahíta Cardona, 1998. Respuesta de la semilla de cedro negro (Juglans neotropica Diels) a la aplicación de tratamientos pregerminativos. Rev. Fac. Nal. Agr. Medellin, 51(1), 217-235.

Masias Bröcker, K., 2007. Caracterización de las propiedades tintóreas del extracto de nogal (Juglans neotropica Diels) proveniente de la cuenca alta del río Zaña. Tesis para optar el Título de Ingeniero Forestal, Facultad de Ciencias Forestales, Universidad Nacional Agraria La Molina; 122 pp. Descargado de http://repositorio.lamolina.edu.pe/bitstream/handle/123456789/82/K50M385-T.pdf? sequence $=1$ \&isAllowed $=y$ en febrero 2014.

Marin, G.C., M.I.R. Mason, H.I. Forde, 1969. Changes in endogenous growth substances in the embryos of Juglans regia during stratification. J. Amer. Soc. Hort. Sci., 94, 13-17.

Mendez, J.M., 2000. Manejo de semillas de 100 especies forestales de América Latina. Danida Forest Seed Centre (DFSC) y Proyecto Semillas Forestales (PROSEFOR). Serie técnica. Manual técnico / CATIE No. 41, 209 pp. CATIE, Turrialba, Costa Rica. Descargado de http://orton.catie.ac.cr/ REPDOC/A4588E/A4588E.PDF en febrero 2014.

Mock, M.M., 2000. Cytokinins: Biosynthesis, metabolism and perception. Vitro Cell. Dev. Biol.-Plant, $36,102-107$. 
Murashige, T., F. Skoog, 1962. A revised médium for rapid growth and bioassys with tobacco tissue cultures. Physiologia Plantarum, 15, 437-497. Descargado de https://essm.tamu. edu/ media/46257/murashigeandskoogintropapersjanick.pdf en 2002.

Pijut, P., 1997. Micropropagation of Juglans cinerea L. (Butternut). Biotechnol. Agric. For., 39, 345357.

Ponce, G., D. Morales, 2011. Estudio de procesos de elaboración de tintes naturales con dos especies vegetales "Nogal" (Junglans neotropica) y "Guarango" (Caesalpinia spinosa) y propuesta de revalorización de saberes ancestrales con las mujeres de la Asociación de Artesanas "Wuarmi Maki" comunidad de Peguche en el Cantón Otavalo. Tesis Pregrado, Facultad de Ingeniería en Ciencias Agropecuarias y Ambientales, Universidad Técnica del Norte (UTN). Descargado de http://repositorio.utn.edu.ec/handle/123456789/823 en enero 2014.

Quintero-García, O. Dario, S. Jaramillo-Villegas, 2012. Rescate y germinación in vitro de embriones inmaduros de cedro negro (Juglans neotropica Diels). Acta Agronómica, 61(1), 52-60. Descargado de $h t t p: / / w w w . r e d a l y c . o r g / p d f / 1699 / 169924335007 . p d f$ en diciembre 2013.

Sánchez-Olate, M., P. Sáez, D. Ríos, 2009. Rhizogenic induction in adult Juglans regia L. cv. Serr tissue induced by Indole Butyric acid and Agrobacterium rhizogenes. CHILEAN JAR, 69(2), 286291.

Toosi, S., K. Dilmagani, 2010. Proliferation of Juglans regia L. by in vitro embryo culture. J. Cell Biol. Genet., 1(1), 12-19. 